heat stress monitoring; and 10) a sample EMS hazardous materials protocol. All equipment and supplies were selected to be easily obtainable from local commercial sources that are specified. The document will be distributed for public comment, following which funding sources will be identified and the program will be implemented by the state.

Conclusions: A document detailing minimum curriculum, operating procedures, and equipment for response to hazardous materials incidents by EMS and emergency department providers has been developed for statewide use.

\section{A Comparison of Infusion Rates of Three Different Infusion Techniques Commonly Used by EMS Personnel}

Jeffrey Keifer, $M D$, Juan March, MD, Kathleen Dunn, $M D$, Lawrence Brown, EMT-P

Division of Emergency Medical Services,

East Carolina University

Purpose: To determine the efficacy of using a standard sphygmomanometer to increase the rate of fluid delivery. Volume replacement with rapid infusion currently is recommended for resuscitation of trauma victims. In the field setting, it is not uncommon to increase the rate of fluid delivery by inflating a sphygmomanometer wrapped around a plastic bag of fluid.

Method: Rate of flow was determined using a $1000 \mathrm{ml}$ plastic bag of normal saline placed at a height of $211 \mathrm{~cm}$ and infused through a standard, large gauge intravenous (IV) tubing with a 14-gauge catheter and vascular resistance assumed to be constant and minimal. Time to delivery of $250 \mathrm{ml}, 500 \mathrm{ml}$, and $750 \mathrm{ml}$ were measured, using only gravity as the control. The study was repeated five times and an average flowrate $(\mathrm{ml} / \mathrm{min})$. In addition, these same measurements were performed using a sphygmomanometer wrapped around the fluid infusion bag and inflated to $300 \mathrm{mmHg}$. The study also was repeated using a commercially available pneumatic pressure bag specifically designed for increasing infusion rates. Flow rate was compared by method and by volume group using analysis of variance (ANOVA).

Results: Mean flow rates \pm standard deviation $(\mathrm{ml} / \mathrm{min})$ Volume from bag $1-250 \mathrm{ml} \quad 251-500 \mathrm{ml} \quad 501-750 \mathrm{ml} p$-value

$\begin{array}{lcccc}\text { Control } & 146 \pm 2 & 140 \pm 6 & 139 \pm 11 & 0.32 \\ \text { BP cuff } & 264 \pm 13 & 156 \pm 4 & 139 \pm 7 & 0.0001 \\ \text { Infuser } & 352 \pm 4 & 245 \pm 6 & 173 \pm 2 & 0.0001\end{array}$

$\begin{array}{llll}p \text {-value } & 0.0001 & 0.0001 & 0.0001\end{array}$

Conclusion: During a resuscitation, a sphygmomanometer can be used to increase flow rates. Intermittent inflation is recommended to maintain maximal flow rate.

\section{Disaster Medicine Fellowship Training}

Clark A. Morres, $M D$

Madigan Army Medical Center

Purpose: To describe a new training program in Disaster Medicine at a military medical center.

Background: United States military involvement in recent disasters worldwide seems certain to continue. Recognizing the important role emergency medicine physicians play in these deployments, we developed a formal training program in Disaster Medicine.

Methods: Madigan Army Medical Center developed a 2-year fellowship in Disaster Medicine in 1993. The curriculum includes completion of didactic training in emergency management, incident command, nuclear and chemical accident management, critical incident stress debriefing, and HAZMAT. Clinical rotations include tropical and preventive medicine, and EMS. The fellow must complete the MPH degree with a disaster epidemiology research project. Field experience is gained in deployments as the commander of a multi-role military disaster response team, and through support to an urban search and rescue task force. Additional first-hand experience is obtained in operational assignments.

Conclusion: Madigan Army Medical Center's Disaster Medicine Fellowship will prepare physicians for military disaster relief missions worldwide.

\section{Emergency Medical Outreach into Communities Following a Disaster: Lessons Learned}

Clark A Morres, MD, Patricia R. Hastings, $M D$

Madigan Army Medical Center

Purpose: To highlight important considerations that will make community outreach successful when delivering medical care during disasters.

Methods: A retrospective analysis of: 1) debriefing records; and 2) after-action reviews of two physicians who provided medical care in a community outreach program one week after the Los Angeles, Calif,, earthquake.

Results: Outreach programs into communities affected by a disaster consist of health professionals providing medical care to victims who are homebound or otherwise unable to seek care. Heath-care providers, such as nonlocal Disaster Medical Assistance Team (DMAT) members, walk from door-to-door, or drive in small teams to see patients isolated by the disaster. This approach offers many potential benefits, particularly when the local infrastructure has been diminished.

Several factors identified in an outreach effort after the Los Angeles earthquake (1994), should be considered when planning disaster outreach missions: 1) clear objectives and limits for the outreach effort must be established; 2) the safety and security of the medical teams and their equipment must be assured to the greatest degree possible; 3) a means of communication must be available; 4) a geographic orientation must be given to each medical team defining the boundaries of the outreach and the planned routes in and out of the area; 5) medical kits should be prepared in accordance with the specific mission objectives; 6 ) a variety of medical contingencies must be anticipated; and 7) plans must be made to provide for urgent evacuation, nonurgent referral, and follow-up care of patients.

Conclusions: Consideration of several important factors will make community outreach a safe and successful medical delivery approach during disasters. 